\title{
Searches for New Physics at the Tevatron and LHC
}

\author{
Peter Wittich ${ }^{a}$, on behalf of the ATLAS, CDF, CMS and D0 Collaborations \\ ${ }^{a}$ Department of Physics, Cornell University, Ithaca, NY 14853, USA, wittich@ cornell .edu
}

\begin{abstract}
This is an auspicious moment in experimental particle physics - there are large data samples at the Tevatron and a new energy regime being explored at the Large Hadron Collider with ever larger data samples. The coincidence of these two events suggests that we will soon be able to address the question, what lies beyond the standard model? Particle physics's current understanding of the universe is embodied in it. The model has been tested to extreme precision - better than a part in ten thousand - but we suspect that it is only an approximation, and that physics beyond this standard model will appear in the data of the Tevatron and LHC in the near future. This brief review touches on the status of searches for new physics at the time of the conference.
\end{abstract}

Keywords: Tevatron, LHC, particle physics, proton-proton collisions, proton-antiproton collisions, collider physics PACS: $12.60 .-\mathrm{i}, 13.85 . \mathrm{Rm}, 14.65 . \mathrm{Jk}, 14.80 .-\mathrm{j}$

The PANIC conference occurred during an exciting time in particle physics. The Tevatron experiments were evaluating results with up to $9 \mathrm{fb}^{-1}$ of data, an amount inconceivable in years past. The LHC had collected its first $1 \mathrm{fb}^{-1}$ of data and results were being presented on datasets 30 times larger than those published in the previous year. As of the time of the conference no evidence for new physics had been seen, but the prospect for discovery is clear in the meantime, the LHC has accumulated more than five times the data set than was presented at the conference, and next year's data set promises to be bigger by another factor of 2-4.

Due to the large number of results that are available, the plenary talk only presented a small number of the results that were available at the time. Many others were shown in the parallel sessions. In addition to the results discussed below, the rest of the results are available on the web [1-4].

This article is organized as follows. After a brief introduction to the experimental facilities, selected results will be recapped with references to more information. We encourage the reader to go to these sources for more information. Due to space constraints very few details can be included in this paper.

\section{Experimental Facilities: Tevatron at FNAL}

The Tevatron is a proton-antiproton collider at the Fermi National Accelerator Laboratory (FNAL), located outside Chicago, Illinois in the US [5]. The Tevatron has been taking data for over 25 years and 2011 was the last year of the proton-antiproton program at Fermilab. During the Run 2 data-taking period, data equivalent to $12 \mathrm{fb}^{-1}$ were $^{-}$ delivered to the experiments. The center-of-mass energy of the collisions in Run 2 is $1.96 \mathrm{TeV}$. The Tevatron has two active interaction points, where general-purpose collider detectors CDF and D0 are housed.

CDF. CDF is a multi-purpose experiment with an emphasis on charged-particle tracking. The CDF II detector is described in detail elsewhere [6]. It has a solenoidal charged particle spectrometer, consisting of 7-8 layers of silicon microstrip detectors and a cylindrical drift chamber immersed in a $1.4 \mathrm{~T}$ solenoidal magnetic field, a segmented sampling calorimeter, and a set of charged particle detectors outside the calorimeter used to identify muon candidates.

DO. The Run 2 D0 detector consists of three major components: a central tracker, liquid argon/uranium calorimeters, and a muon spectrometer. The tracking system consists of a silicon strip tracker and a fiber tracker. Both are enclosed in a $2 \mathrm{~T}$ solenoidal magnetic field. The muon system consists of gaseous detectors with toroidal magnetic fields to assist in the momentum measurement. More details can be found elsewhere [7]. 

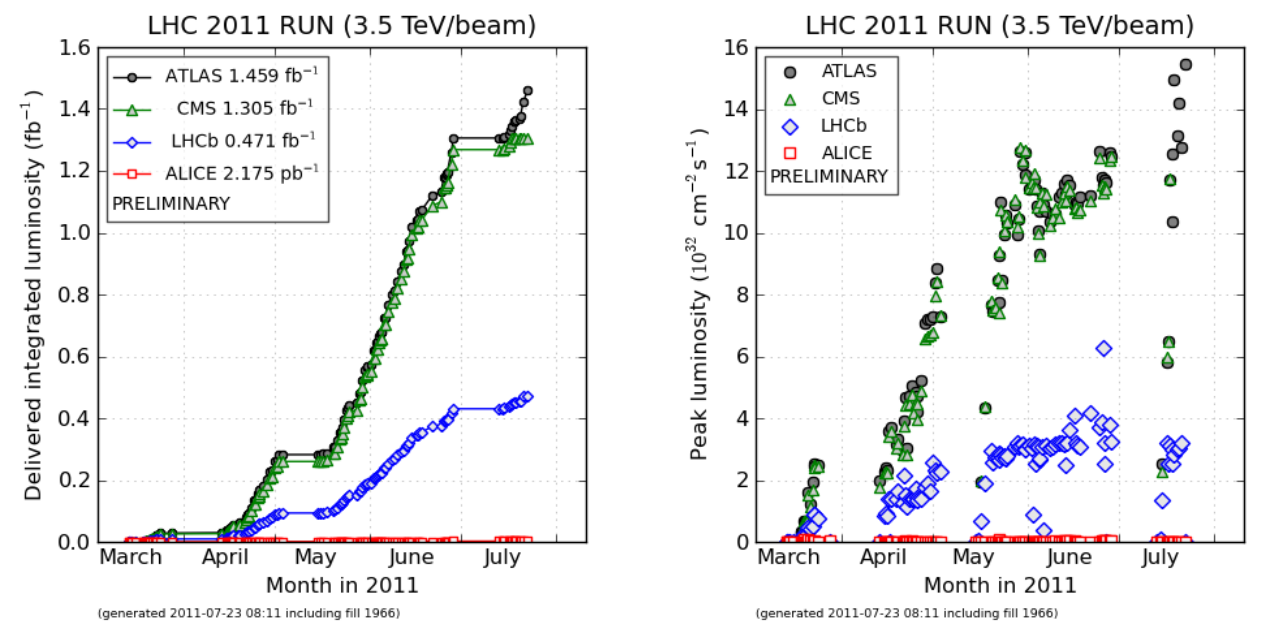

FIGURE 1. LHC luminosity as a function of calendar day at the time of the PANIC11 conference. Different colors represent different interaction regions at the LHC. The luminosity at ALICE and LHCb is not optimized for the overall largest luminosity.

\section{Experimental Facilities: CERN LHC}

The Large Hadron Collider (LHC) is a proton-proton collider at the CERN Laboratory in Geneva, Switzerland [8]. There are four collision regions at the LHC. Two of the four are occupied by the general-purpose experiments, CMS and ATLAS. The other two are occupied by LHCb, an experiment dedicated to studying the production and decays of $B$ hadrons and mesons, and ALICE, an experiment dedicated to heavy-ion collisions. Compared to the Tevatron experiments, ATLAS and CMS are next-generation detectors. Their fiducial coverage extends further in $\eta$, and most systems have higher segmentation to handle the more demanding environment of the LHC. At design performance, the LHC will collide bunches every $25 \mathrm{~ns}$ (compared to $396 \mathrm{~ns}$ for the Tevatron) at an energy of $\sqrt{s}=14 \mathrm{TeV}$ (compared to just under $2 \mathrm{TeV}$ at Fermilab.) The higher bunch intensities will lead to more than five times higher pileup, i.e., five times higher number of extra interactions per crossing, as well, and the overall design instantaneous luminosity of $10^{34} / \mathrm{cm}^{2} / \mathrm{s}$ is also 25 times higher than the maximum achieved at Fermilab.

The current data-taking period started in December 2009. In the calendar year 2010, 35/pb of data were collected at a center-of-mass energy of $7 \mathrm{TeV}$; at the time of PANIC11, more than $1 \mathrm{fb}^{-1}$ had been collected in 2011 at the same energy. Figure 1 shows the accumulation of data as a function of time as well as the highest recorded instantaneous luminosity at the time of the conference (roughly $1.6 \times 10^{33} / \mathrm{cm}^{2} / \mathrm{s}$ ). More than $45 / \mathrm{pb}$ of data were being accumulated per day. In the meantime, instantaneous luminosities as high as $3.7 \times 10^{33} / \mathrm{cm}^{2} / \mathrm{s}$ have been recorded, and more than $130 / \mathrm{pb}$ of data have been accumulated in a single store (which is roughly equivalent to a day). For the calendar year 2011, the LHC goal was to deliver $1 \mathrm{fb}^{-1}$ of data. This goal was already achieved by the time of the conference, and more than $5 \mathrm{fb}^{-1}$ of data have been delivered in 2011.

CMS. The Compact Muon Solenoid (CMS) detector is one of two large general-purpose experiments at the LHC. The 12,500 ton detector features nearly complete solid-angle coverage and can precisely measure electrons, photons, muons, jets and missing energy over a large range of particle energies. A detailed description of the CMS detector can be found elsewhere [9]. The detector consists of the pixel and silicon strip trackers. They provide coverage in the region $|\eta|<2.5$ and are immersed in a $3.8 \mathrm{~T}$ magnetic field to allow momentum determination of charged particles. The electromagnetic and hadron calorimeters are used to detect energy deposits from electrons in the range $|\eta|<2.5$ as well as to provide an estimate of missing transverse energy due to escaping particles. The electromagnetic calorimeter has an energy resolution of better than $0.5 \%$ for unconverted photons with transverse energies above $100 \mathrm{GeV}$. A set of gaseous detectors outside the solenoids is used to detect the passage of minimum-ionizing particles, mainly muons.

ATLAS. The other large general-purpose detector is ATLAS. ATLAS is described in detail elsewhere [10]. The tracking system consists of a silicon pixel detector, a silicon strip tracker, a straw chamber with transition radiation particle ID. The tracker is bathed in a $2 \mathrm{~T}$ solenoidal magnetic field. Outside of the magnetic field, electromagnetic 
and hadronic calorimeters allow measurement of the energy deposited by passing particles. There are three toriodal magnetic fields (two in the endcaps, one in the central region) that produce magnetic fields for the muon systems of $1 \mathrm{~T}$ and $0.5 \mathrm{~T}$, respectively. The muon system consists of separate high-precision tracking chambers and triggering system, with the goal of achieving $10 \%$ resolution for $1 \mathrm{TeV}$ muons.

\section{SEARCHES FOR NEW PHYSICS}

The program of searches for new physics at collider experiments is exhaustive. We look for new physics either in direct or in indirect searches. In direct searches, the goal is to look for evidence of on-shell production of massive undiscovered particles. These particles can be strongly produced and can decay either strongly, to hadronic final states, or weakly, to leptonic final states. A more complicated possibility includes some strong and some weak decays in complicated decays when multiple new particles are produced, leading to mixed hadronic and leptonic final states.

In indirect searches, rather than look for the direct production of a heavy new particle, we make precision measurements of quantities that can be affected by the presence of new particles, e.g. through interference. These effects often come through loop diagrams, where one internal line in a loop is replaced by a new particle. The presence of the new particle changes the measured quantity, such as a branching ratio, from its standard model expectation. Since the new particles that are produced are typically off-shell, these searches are able to probe much higher masses than the direct searches. However, they are dependent on the theoretical calculations and good understanding of systematic uncertainties.

Direct searches can be characterized by the final state particles. Many of the most sensitive early searches at the LHC involve strongly produced particles (which have large cross sections) that decay to hadronic final states (with large branching fractions.) The challenge with these searches is to suppress the equally large multi-jet background. Some of these searches are discussed below, including narrow dijet resonances, jets $+E_{T}$ SUSY searches, and searches for SUSY in events with $b$ jets and $E_{T}$.
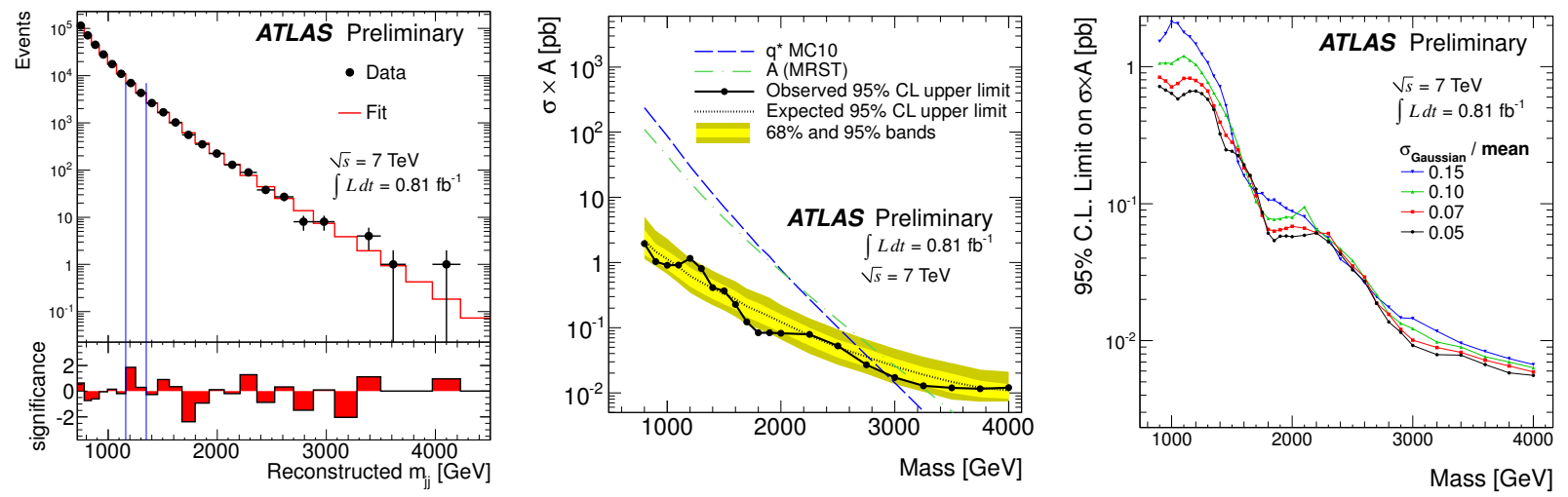

FIGURE 2. ATLAS results for searches for narrow dijet resonances in $0.81 \mathrm{fb}^{-1}$ of data. Left: the invariant mass spectrum of pairs of jets selected by this analysis. No significant excess is observed. Middle: limit curves for $\sigma \times A$ for excited quark models. Right: limit curves for $\sigma \times A$ for simple Gaussian resonances decaying to dijets as a function of mass, for values of the fractional Gaussian width.

Narrow resonances in dijet events. For the conference, ATLAS presented a new search for resonant dijet production of new particles. Using $0.81 \mathrm{fb}^{-1}$ of data, the ATLAS collaboration calculates the invariant mass of pairs of jets (see Fig. 2 left) and looks for structure in the falling spectrum indicative of resonant $s$-channel production of new particles (e.g., $g g \rightarrow X \rightarrow q q$ ) [11]. These searches are sensitive to many new physics models such as excited quarks and contact interactions, and are also sensitive to quark substructure, and are therefore the modern equivalent of Rutherford scattering - relevant since the conference was dedicated to the Rutherford centennial. No significant excess was observed, and the collaboration presented the results as limits on the cross section times branching ratio times acceptance for both excited quarks (Fig. 2 middle) and for simple parameterized Gaussian shapes (Fig. 2 right.) In the latter case, the results are presented as a function of the mean of the Gaussian for several fractional widths (5-15\%). Using these generic assumptions, limits are placed in the range of $1-10^{-2} \mathrm{pb}$ for masses in the range of $1-3 \mathrm{TeV} / \mathrm{c}^{2}$. CMS has released similar results [12]. 
Squarks and Gluinos. In supersymmetric models, $\tilde{g} \tilde{g}, \tilde{g} \tilde{q}$ and $\tilde{q} \tilde{q}$ production leads to hadronic final states. If $R$ parity is assumed to be conserved, the lightest supersymmetric particle is stable and usually assumed to be the dark matter candidate, i.e., neutral. In this case, the final state contains missing transverse momentum $\left(E_{T}\right)^{1}$ due to the escaping undetected particles. As such the final state consists of jets and large $E_{T}$. The analysis uses $1 \mathrm{fb}^{-1}$ of data collected using a trigger requiring a jet with $E_{T}>75 \mathrm{GeV}$ and $E_{T}>45 \mathrm{GeV}$. The measurement is optimized for different squark and gluino final states, and the final number of event counts is extracted by a likelihood fit to the a mass-like variable $\left(m_{\text {eff }}\right)$, which is the sum of $E_{T}$ and the magnitudes of the transverse momenta of the highest momentum jets considered in the analysis. No excess above SM expectation is observed, and limits are set in two ways: an mSUGRA scenario, and a simplified model where the only free parameters are the masses of the squarks and gluinos and the LSP mass is assumed to be zero. The results can be seen in Fig. 3. In the simplified models,
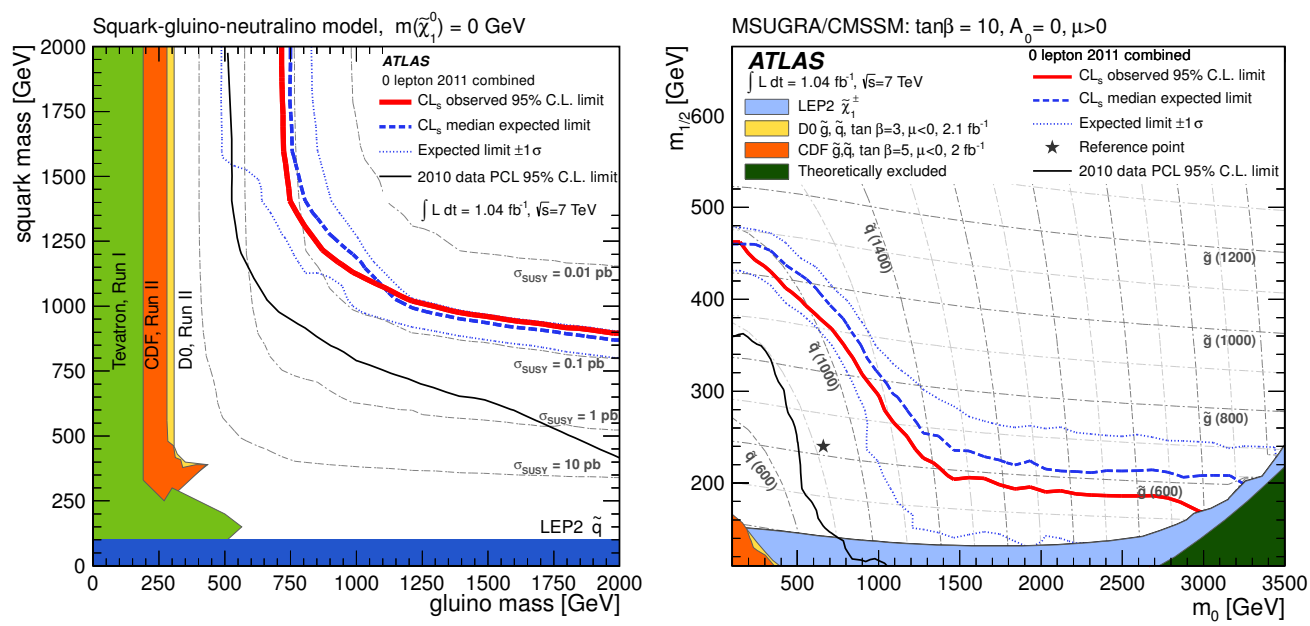

FIGURE 3. ATLAS: Interpretation of null results for searches for new physics with jets $+E_{T}$ in a simplified model (left) and mSugra (right). In the simplified models, gluino (squark) masses below $700(875) \mathrm{GeV} / c^{2}$ are excluded at the $95 \%$ confidence level, while in the mSugra-inspired model squarks and gluinos of equal mass are disallowed for masses below $950 \mathrm{GeV} / c^{2}(\tan \beta=10$, $A_{0}=0$ and $\left.\mu>0\right)$.

gluino (squark) masses below $700(875) \mathrm{GeV} / c^{2}$ are excluded at the $95 \%$ confidence level, while in the mSUGRAinspired model squarks and gluinos of equal mass are disallowed for masses below $950 \mathrm{GeV} / c^{2}\left(\tan \beta=10, A_{0}=0\right.$ and $\mu>0)$ [13].

Figure 4 shows the result of a CMS search targeting a similar final state [14]. The measurement uses a discriminating variable called $\alpha_{T}$ to suppress the multi-jet background. The variable is defined as $\alpha_{T} \equiv E_{T}^{\mathrm{jet} 2} / M_{T}^{1,2}$, where $E_{T}^{\mathrm{jet} 2}$ is the transverse energy of the subleading jet and $M_{T}^{1,2}$ is the transverse mass using the first two jets, and events are required to pass $\alpha_{T}>0.55$. This variable is very effective at suppressing QCD multi-jet backgrounds, as can be seen in Fig. 4 left. Figure 4 right shows the result of this search using $1.1 \mathrm{fb}^{-1}$ of data. Using an constrained SUSY model (CMSSM) [15] to set limits, squark and gluino masses of $1.1 \mathrm{TeV}$ are excluded for $m_{0}<0.5 \mathrm{TeV}$.

In supersymmetric models, the third family is special. The top squarks can be lighter than other squarks due to the large mass of the top quark. To a lesser extent, the bottom squark might be much lighter than the other squarks. Therefore, $\tilde{t}_{1}$ or $\tilde{b}_{1}$ could be produced copiously at the LHC, either directly or via decays of gluinos The ATLAS collaboration has mounted a search for $b$ squarks with $0.83 \mathrm{fb}^{-1}$ of data [16]. They consider either the case of direct production or decays through intermediate gluinos (e.g. $\tilde{g} \rightarrow \tilde{b}_{1} b$ ), where $\tilde{b}_{1} \rightarrow b \tilde{\chi}_{1}^{0}$. The analysis requires events with at least one jet with $p_{T}>130 \mathrm{GeV} / c$, an additional jet with $p_{T}>50 \mathrm{GeV} / c$ and $E_{T}>130 \mathrm{GeV}$. The displacement of the common vertex of tracks associated with a jet with respect to the interaction point is used to identify $b$ jets. Events with identified leptons are vetoed. There is no evidence for an excess beyond the SM expectations. Results on are shown in Fig. 5 as a function of the gluino mass and bottom squark mass, with the assumption $m_{\tilde{\chi}_{1}^{0}}=60 \mathrm{GeV} / c^{2}$. Gluinos up to a mass of $600 \mathrm{GeV} / c^{2}$ are forbidden up to $\tilde{b}$ masses at the kinematic limit. As can be seen from Fig. 5,

\footnotetext{
${ }^{1}$ We define the missing transverse momentum $\vec{E}_{T} \equiv-\sum_{i} E_{\mathrm{T}}^{i} \mathbf{n}_{i}$, where $\mathbf{n}_{i}$ is the unit vector in the azimuthal plane that points from the beamline to the $i$ th calorimeter tower. We call the magnitude of this vector $E_{T}$.
} 

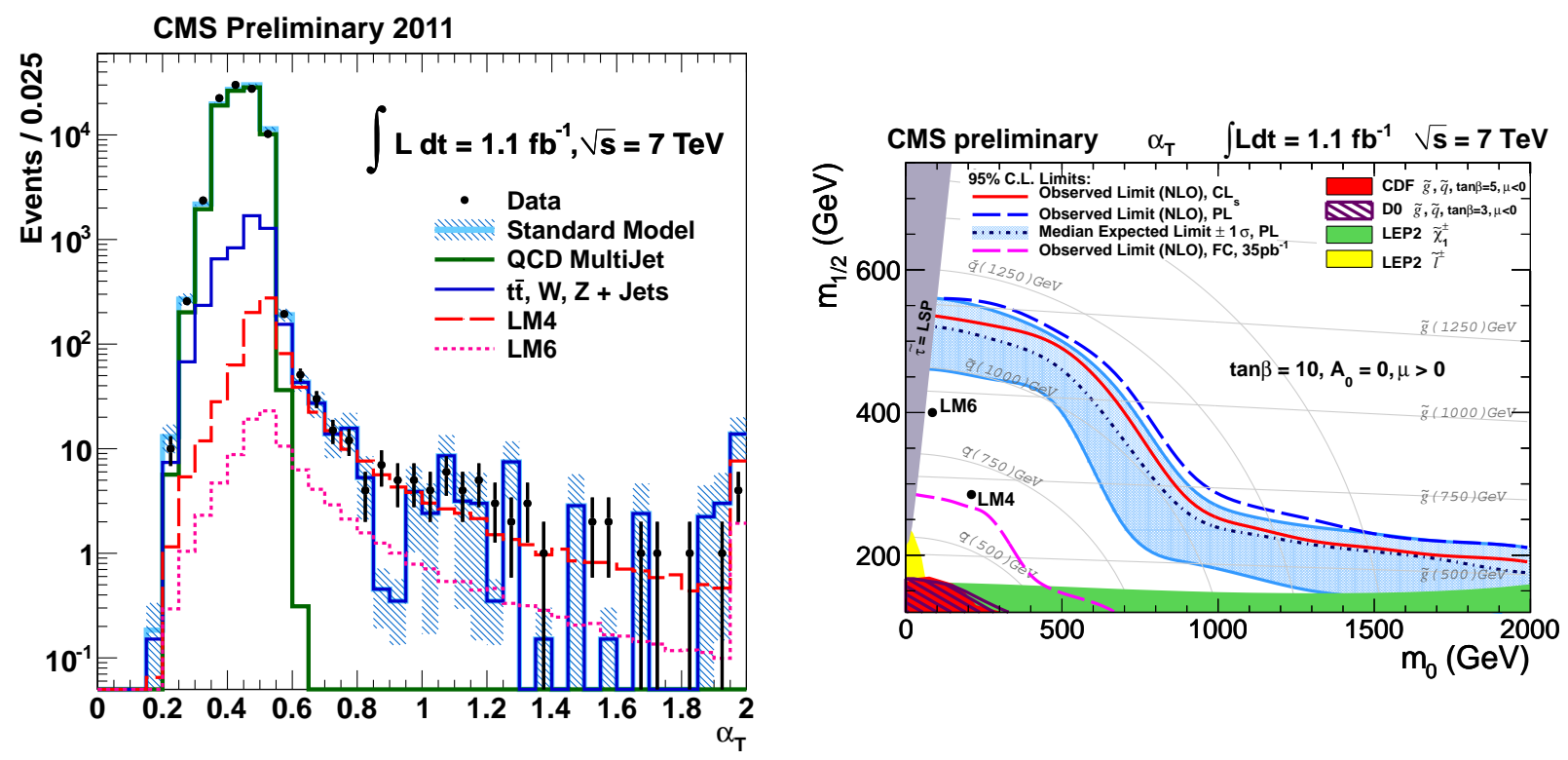

FIGURE 4. CMS search for SUSY in hadronic final states using $\alpha_{T}$. Left: Comparison of $\alpha_{T}$ distribution with data and expectation. The strong rejection power of $\alpha_{T}$ against QCD multi-jet backgrounds is apparent from the distribution, before the $\alpha_{T}>0.55$ requirement is applied. Right: The null result is interpreted in terms of CMSSM SUSY model. Squark and gluino masses of $1.1 \mathrm{TeV} / c^{2}$ are excluded for $m_{0}<0.5 \mathrm{TeV} / c^{2}$.

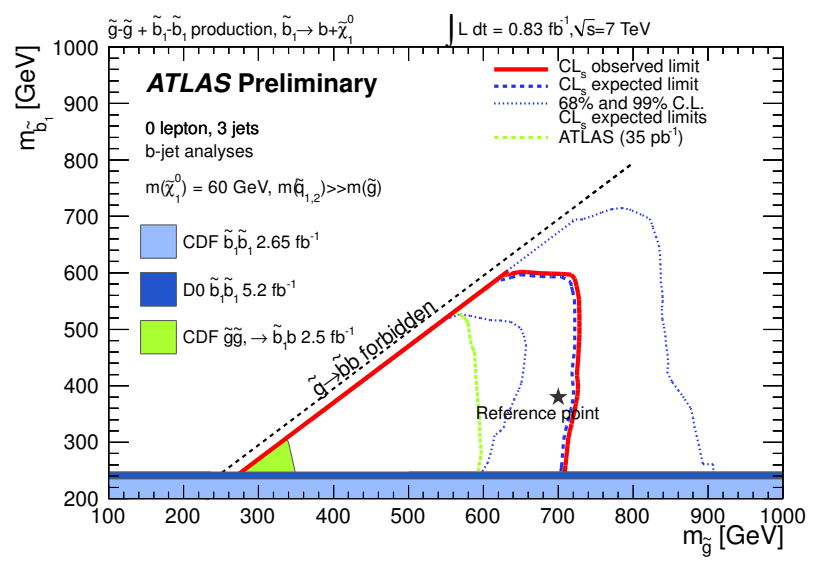

FIGURE 5. ATLAS results for searches for new physics with $b$ jets $+E_{T}$ using $0.83 \mathrm{fb}^{-1}$ of LHC data. Shown is the excluded region for gluino and $b$ squark pair production followed by the decays $\tilde{g} \rightarrow \tilde{b}_{1} b$ and $\tilde{b}_{1} \rightarrow b \tilde{\chi}_{1}^{0}$, under the assumption $m_{\tilde{\chi}_{1}^{0}}=$ $60 \mathrm{GeV} / c^{2}$. The limits obtained in this measurement extend the reach of experiments much beyond the shown Tevatron contours.

the limits extend much beyond the shown Tevatron limits with the same model assumptions.

Searches for new gauge bosons. Searches for new gauge bosons have been a staple at the Tevatron for many years. $Z^{\prime}$ bosons can be found by looking for narrow dilepton resonances. The CMS collaboration has searched for narrow di-electron and di-muon resonances with data corresponding to $1.1 \mathrm{fb}^{-1}$ of data. No signal beyond SM background is observed, and the results are interpreted in terms of SM-like $Z^{\prime}$ gauge bosons, Kaluza-Klein gravitons and superstring inspired models. SM-like $Z^{\prime}$ s are excluded for $m<1940 \mathrm{GeV} / c^{2}$, as can be seen in Fig 6 [17].

Searches for heavy copies of the $W$ gauge boson have also broken new ground. At the time of the conference, the main experimental $W^{\prime}$ searches from the LHC were in the leptonic decay mode: $W^{\prime} \rightarrow \ell v$, where $\ell=\mu$ or $e$. Using $1.1 \mathrm{fb}^{-1}$ of data, the transverse mass distribution is examined in events with a single isolated high-momentum lepton 

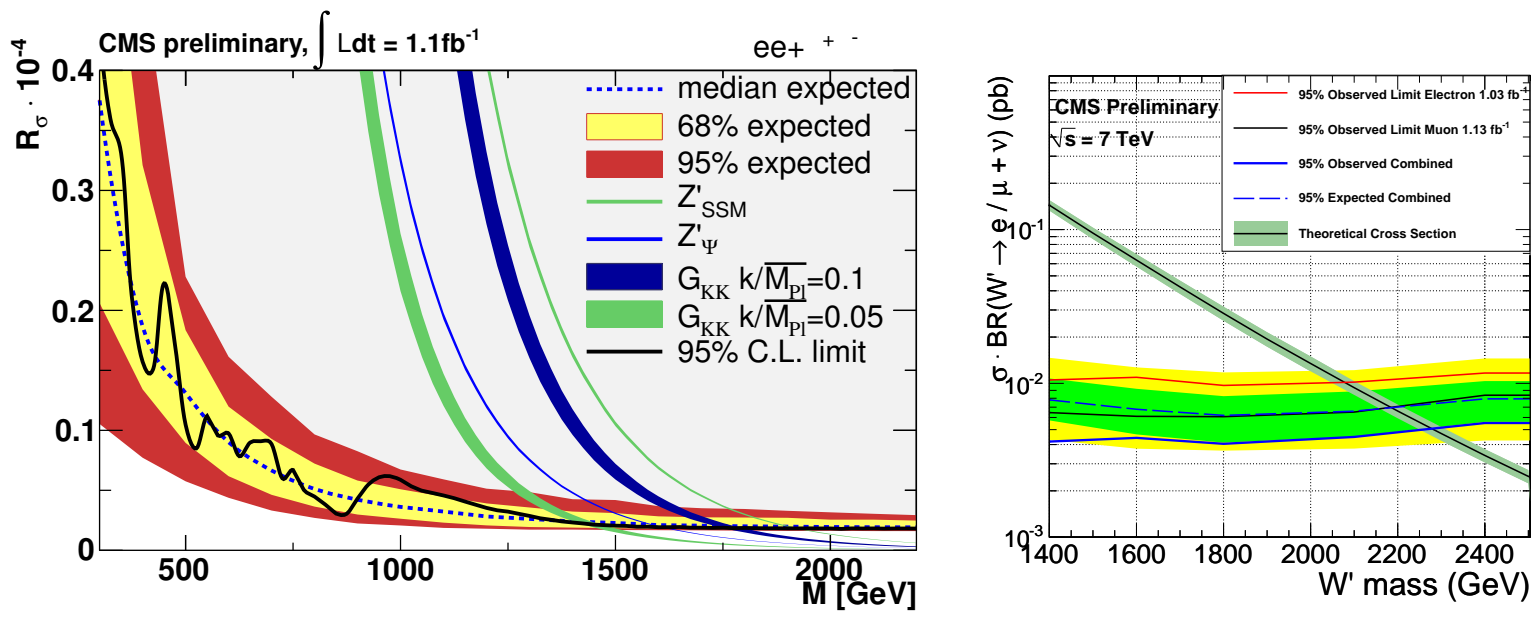

FIGURE 6. Left: limits on production of narrow resonances that decay to di-muon or di-electron pairs, such as $Z^{\prime} \rightarrow \ell \ell$, from the CMS experiment. No excess is observed. SM-like heavy new $Z$ gauge bosons are excluded for $m<1940 \mathrm{GeV} / c^{2}$. Right: Limits on $\sigma \times B R\left(W^{\prime} \rightarrow \ell v\right)$ from a null result from CMS. Limits are placed on SM-like $W^{\prime}$ for $m<2.27 \mathrm{TeV} / c^{2}$.
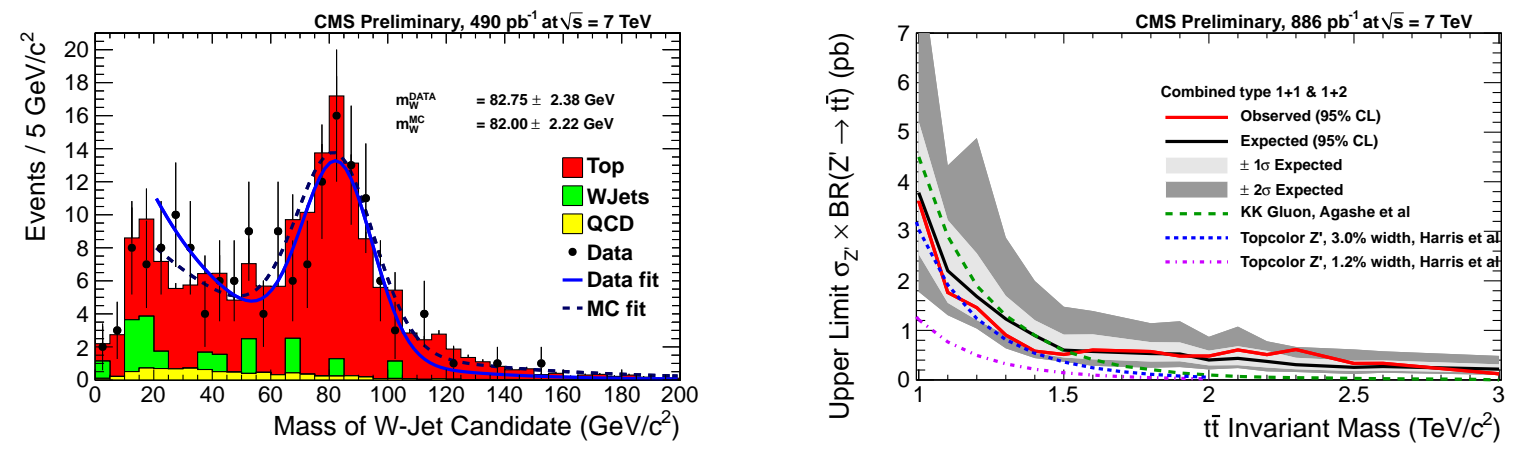

FIGURE 7. Left: Mass of the highest mass jet in a semileptonic top sample, demonstrating the jet substructure technique. Right: limits on $\sigma \times \mathscr{B}\left(Z^{\prime} \rightarrow t \bar{t}\right)$, in a CMS $Z^{\prime}$ search.

and large missing energy for an excess that would signal the decay of a $W^{\prime}$. No excess is found, and heavy boson masses below $2.27 \mathrm{TeV}$ are excluded at the 95\% confidence level, assuming standard model-like couplings [18]. Figure 6 shows the limits for both $W^{\prime}$ and $Z^{\prime}$. Similar results were obtained by the ATLAS collaboration [19-21].

New gauge bosons can of course also decay in other ways. One search presented at the conference uses new techniques developed in collaboration between the theoretical and experimental communities that focus on the details of the structure of energy deposits within jets, originally proposed in conjunction with $V H$ searches at the LHC [22]. The paper proposed selecting very boosted Higgs bosons such that the decay products of the Higgs are reconstructed as one jet with sub-jets, rather than as two distinct jets, and an algorithm for detecting such jet substructure. Since then many more techniques based on jet sub- and superstructure have been proposed, including those to detect boosted top quarks ("top taggers"), where the $t \rightarrow W b$ decay products are not reconstructed as distinct objects. The CMS collaboration has performed a search for $Z^{\prime} \rightarrow t \bar{t}$ process where the top quarks are highly boosted and therefore the decay products of one or both of the top quarks is collimated into one jet ( $b$ quark, both hadronic $W$ decay products in one jet) or two jets (both hadronic $W$ decay products in one jet), rather than the usual three jets [23]. The use of this technique extends the sensitivity of the search to higher $Z^{\prime}$ masses. Figure 7 left demonstrates the ability of the top taggers to find $W$ bosons in a top-quark enriched data sample. No excess is observed beyond SM expectation. Analyzing $0.865 \mathrm{fb}^{-1}$ of data, CMS sets a limit on $\sigma_{Z^{\prime}} \times \mathscr{B}\left(Z^{\prime} \rightarrow t \bar{t}\right)<1 \mathrm{pb}$ for $m<1.1 \mathrm{TeV}$, as shown in Fig. 7 right.

$\mathrm{CDF}$ has reported an excess in the invariant mass spectrum of jets in events with leptonically decaying $W$ bosons [24]. In $4.3 \mathrm{fb}^{-1}$ of data, the collaboration reported an excess in the range $120-160 \mathrm{GeV} / c^{2}$. The excess was 

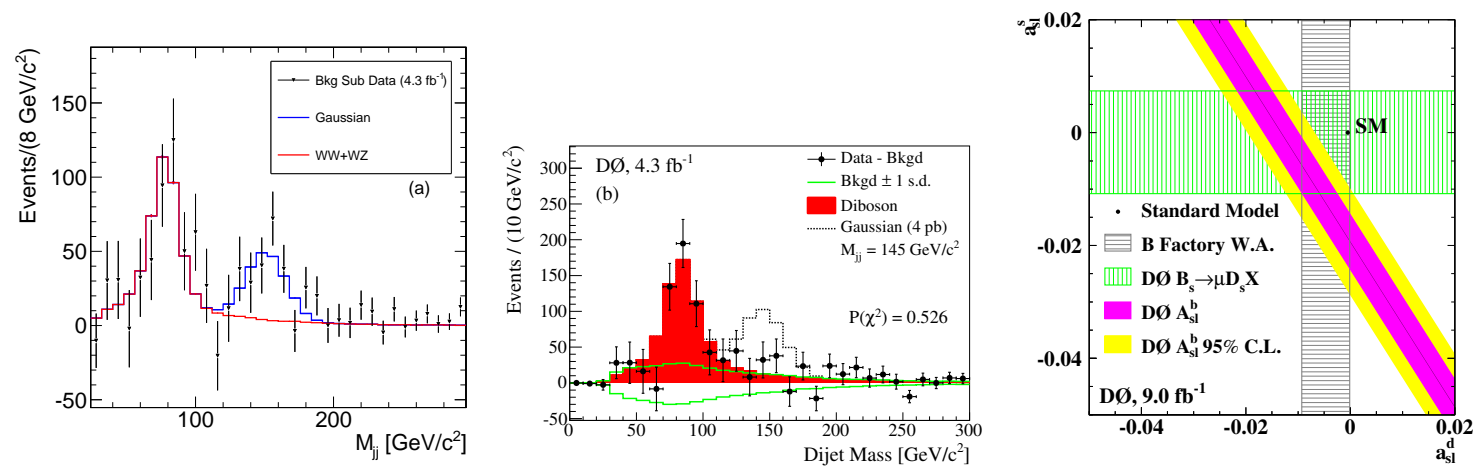

(a) $\mathrm{CDF}$ and $\mathrm{D} 0 \mathrm{~W}+$ jet events.

(b) D0 Like-sign dimuon asymmetry.

FIGURE 8. Left and middle: Background-subtracted invariant mass distribution of two jets from a sample of $W+$ jet events. Left: CDF. The blue line shows a Gaussian approximation of a new physics signal with a resolution compatible with the detector resolution and a cross section of approximately three pb. Middle: D0. A Gaussian signal with a 4 pb signal is superposed. No evidence for a non-standard signal is observed and a CDF-like anomaly is ruled out at 2.5 standard deviations. Right: Likesign dimuon asymmetry measured in semi-leptonic $B$ meson decays at the D0 experiment in $9 \mathrm{fb}^{-1}$ of data. The asymmetry is incompatible with the standard model at $3.9 \sigma$.

deemed incompatible with the standard model expectations after taking all known statistical and systematic effects into account. In a conference result, CDF announced a result with an increased dataset $\left(7.3 \mathrm{fb}^{-1}\right)$ [25] the significance of the excess increased from 3.2 to 4.1 standard deviations. Figure 8(a) shows the excess from the publication, as well the result from D0 [26]. Analyzing the same amount of data as the CDF publication $\left(4.3 \mathrm{fb}^{-1}\right)$ the D0 analysis checks the CDF measurement and rules out a CDF-like excess at more than three standard deviations. At the LHC, ATLAS has also weighed in and cannot confirm the CDF result either [27]. At this point it is unclear what the origin of the difference between the CDF and D0 results is, though the collaborations have released a statement that the data of the two experiments is compatible with each other within 2.5 standard deviations.

$A_{f b}$ in $t \bar{t}$ production. $\mathrm{CDF}$ and D0 have measured the forward-backward asymmetry in top quark pair production. In the Tevatron, the anti-top is produced mostly along the direction of the anti-proton beam with a small asymmetry predicted by the SM. Both CDF and D0 have tried to measure this asymmetry in the past and seen a high value. $\mathrm{CDF}$ reports that the effect is seen to increase as a function of the invariant mass of the $t \bar{t}$ system and the rapidity difference [28]. For $m_{t \bar{t}}>450 \mathrm{GeV} / c^{2}$, the parton-level asymmetry is measured to be $A^{t \bar{t}}=0.475 \pm 0.114$ compared to a next-to-leading order QCD prediction of $0.088 \pm 0.013$. D0 has measured a similar though less significant excess in $5.4 \mathrm{fb}^{-1}$ of data [29].

At a $p p$ machine like the LHC, there is no intrinsic asymmetry in the standard model. The forward-backward asymmetry manifests itself as a discrepancy in the number of forward vs central top quark pairs [30]. However, estimates suggest that $60 \mathrm{fb}^{-1}$ are required to allow the LHC to test this.

Anomalous like-sign dimuon charge asymmetry. D0 has reported an excess in the number of same-sign muon pairs in semihadronic $B$ decays using $9 \mathrm{fb}^{-1}$ of data [31]. D0 counts the difference between the number of events with two same-sign positive and negative muons, $A_{\mathrm{sl}}^{b} \equiv\left(N_{b}^{++}-N_{b}^{--}\right) /\left(N_{b}^{++}+N_{b}^{--}\right)$, which can be related to the quark-level asymmetries $a_{\mathrm{sl}}^{d}$ and $a_{\mathrm{sl}}^{s}$. They find an excess compared to the SM prediction:

$$
\begin{aligned}
A_{s l}^{b} & =[-0.787 \pm 0.172(\text { stat }) \pm 0.093(\text { syst })] \% \\
A_{s l}^{b}(\mathrm{SM}) & =\left[-0.028_{-0.006}^{+0.005}\right] \%
\end{aligned}
$$

The discrepancy is about $3.9 \sigma$. Figure 8(b) shows this result compared to SM predictions in terms of the quark-level asymmetries. 


\section{CONCLUSION}

The PANIC2011 conference took place during an exciting time for particle physics. Tevatron results with $9 \mathrm{fb}^{-1}$ of data were presented, showing some interesting hints that the LHC will be able to follow up on. LHC results with thirty times more statistics than previously available were also shown. Since then, the LHC has collected another factor of five times more data. While no evidence for new physics has yet to be discovered, it is likely that the next year will be very interesting. As mentioned in the introduction, this brief report only allows an overview of a small number of the results available from ATLAS, CDF, CMS and D0. Many more results from all experiments listed are available on the web [1-4].

\section{ACKNOWLEDGMENTS}

We would like to thank the collaborations for their help and the accelerator divisons of CERN and FNAL for their great work. Copyright CERN on behalf of the ATLAS and CMS Collaborations.

\section{REFERENCES}

1. ATLAS public physics results (2011), URL https://twiki.cern. ch/twiki/bin/view/AtlasPublic.

2. CMS public physics results (2011), URL https://twiki.cern.ch/twiki/bin/view/CMSPublic/ PhysicsResults.

3. CDF public physics results (2011), URL http://www-cdf.fnal.gov/physics/physics.html.

4. D0 public physics results (2011), URL http://www-d0.fnal.gov/results/index.html.

5. R. R. Wilson, Phys.Today 30N10, 23-30 (1977).

6. CDF Collaboration, A. Abulencia, et al., J. Phys. G 34, 2457 (2007).

7. D0 Collaboration, V. Abazov, et al., Nucl. Instr. Methods A 565, 463 (2006).

8. L. Evans, and P. Bryant, JINST 3, S08001 (2008).

9. CMS Collaboration, S. Chatrchyan, et al., JINST 3, S08004 (2008).

10. ATLAS Collaboration, G. Aad, et al., JINST 3, S08003 (2008).

11. ATLAS Collaboration, Search for new physics in dijet mass distributions in $0.81 \mathrm{fb}^{-1}$ of $p p$ collisions at $\sqrt{s}=7 \mathrm{TeV}(2011)$, ATLAS-CONF-2011-095.

12. CMS Collaboration, S. Chatrchyan, et al. (2011), arXiv:1107. 4771.

13. ATLAS Collaboration, G. Aad, et al. (2011), submitted to Phys. Lett. B, arXiv:1109. 6572.

14. CMS Collaboration, S. Chatrchyan, et al. (2011), arXiv:1109.2352v1.

15. G. L. Kane, C. Kolda, L. Roszkowski, and J. D. Wells, Phys. Rev. D 49, 6173 (1994).

16. ATLAS Collaboration, Search for supersymmetry in $p p$ collisions at $\sqrt{s}=7 \mathrm{TeV}$ in final states with missing transverse momentum, b-jets and no leptons with the ATLAS detector (2011), ATLAS-CONF-2011-098.

17. CMS Collaboration, Search for resonances in the dilepton mass distribution in $p p$ collisions at $\sqrt{s}=7 \mathrm{TeV}(2011)$, CMS-PAS-EXO-11-019.

18. CMS Collaboration, Search for $W^{\prime}$ in the leptonic channels in $p p$ collisions at $\sqrt{s}=7 \mathrm{TeV}$ (2011), CMS-PAS-EXO-11-024.

19. ATLAS Collaboration, G. Aad, et al. (2011), arXiv:1108.1582.

20. ATLAS Collaboration, Search for a heavy neutral particle decaying into an electron and a muon pair using $\mathrm{L}=0.87 \mathrm{fb}^{-1} \mathrm{of}$ ATLAS data (2011), ATLAS-CONF-2011-109.

21. ATLAS Collaboration, G. Aad, et al., Phys. Lett. B705, 28-46 (2011).

22. J. M. Butterworth, A. R. Davison, M. Rubin, and G. P. Salam, Phys. Rev. Lett. 100, 242001 (2008).

23. CMS Collaboration, Search for BSM $t \bar{t}$ production in the boosted all-hadronic final state (2011), CMS-PAS-EXO-11-006.

24. CDF Collaboration, T. Aaltonen, et al., Phys. Rev. Lett. 106, 171801 (2011).

25. CDF Collaboration, $W j j$ update to $7 \mathrm{fb}^{-1}$ (2011), URL http://www-cdf. fnal.gov/physics/ewk/2011/wjj/ 7_3.html.

26. D0 Collaboration, V. M. Abazov, et al., Phys Rev. Lett. 107, 011804 (2011).

27. ATLAS Collaboration, Invariant mass distribution of jet pairs produced in association with a leptonically decaying $W$ boson using $1.02 \mathrm{fb}^{-1}$ of ATLAS data (2011), ATLAS-CONF-2011-097.

28. CDF Collaboration, T. Aaltonen, et al., Phys. Rev. D 83, 112003 (2010).

29. D0 Collaboration, V. M. Abazov, et al. (2011), arXiv: 1107.4995.

30. J. L. Hewett, J. Shelton, M. Spannowsky, T. M. P. Tait, and M. Takeuchi (2011), arXiv: 1101.0034.

31. D0 Collaboration, V. M. Abazov, et al., Phys.Rev. D 84, 052007 (2011). 